
3

\title{
Polysaccharide of Atractylodes Macrocephala Koidz(PAMK) Enhance the Immunomodulatory Activity of RAW264.7 Through TLR4/MyD88/NF-kB Pathway
}

\section{Wanyan Li}

Zhongkai University of Agriculture and Engineering

\section{Xuelian Xiang}

Zhongkai University of Agriculture and Engineering

Nan Cao

Zhongkai University of Agriculture and Engineering

\section{Yunbo Tian}

Zhongkai University of Agriculture and Engineering

\section{Wenbin Chen}

Zhongkai University of Agriculture and Engineering

\section{Xumeng Zhang}

Zhongkai University of Agriculture and Engineering

Wenjun Liu

Zhongkai University of Agriculture and Engineering

\section{Xinliang Fu}

Zhongkai University of Agriculture and Engineering

Jia Liu

Jilin Engineering Normal University

Danning Xu ( $\nabla$ xdanning@126.com )

Zhongkai University of Agriculture and Engineering

\section{Research Article}

Keywords: PAMK, RAW264.7, TLR4/NF-KB pathway

Posted Date: April 27th, 2021

DOl: https://doi.org/10.21203/rs.3.rs-426692/v1

License: (9) This work is licensed under a Creative Commons Attribution 4.0 International License.

Read Full License 
Page 2/15 


\section{Abstract}

The Polysaccharide of Atractylodes macrocephala Koidz (PAMK) has been reported to play an important role in improving the immune function of mammals and poultry. But the molecular mechanism in cell is still not unclear. For investigating the mechanism in mice, RAW264.7 were cultured and divided into six groups (cell, NC, siTLR4, NC + PAMK, PAMK, siTLR4 + PAMK). The serum cytokines, mRNA relative expression level of cytokines and TLR4 signal pathway, the protein expression level of TLR4 signal pathway were measured. The results showed PAMK could increase the level of IL-4, IL-6, TNF- $a$, IFN- $\gamma$, and increase the mRNA relative expression level and protein expression level of TLR4, MyD88, IKK, NF-KB. In conclusion, PAMK may regulate the immue activity of RAW264.7 through the TLR4/MyD88/NF-KB pathway.

\section{Introduction}

Polysaccharide of Atractylodes macrocephala Koidz (PAMK) as one of the main extracts of Atractylodes macrocephala, which is a traditional medicine and has been utilized for at least 2000 years. PAMK was often used to treat diarrhea, spleen and stomach disease, respiratory infection etc ${ }^{1}$. PAMK has antiinflammatory, anti-cancer activities, maintains the balance of gastrointestinal microbiome and regulates the organism's immunity balance ${ }^{1}$. In addition, PAMK promotes the proliferation of lymphocytes which in spleen and thymus of human, chicken, and mice, cause it could induce T lymphocytes enter S and G2/M phases, and effectively elevates the percentage of CD $4+$ and CD $8+\mathrm{T}$ cells ${ }^{4}$. PAMK also increases the index of spleen and thymus in geese, upregulates the level of interleukin-2 (IL-2), interleukin-4 (IL-4), interleukin-5 (IL-5), interleukin-6 (IL-6), interferon-y (IFN- - ), Immunoglobulin G (IgG) in serum of mice and geese $^{5} ;{ }^{6}$. However, $\mathrm{Xu}^{7}$ indicated that PAMK could decrease the high level of Tumor Necrosis Factor-a (TNF- $a$ ), interleukin-1 (IL-1), $\mathrm{H}_{2} \mathrm{O}_{2}$ caused by heat stress (HS) in geese. Jeong $D^{8}$. suggested that PAMK down regulated the high level of pro-inflammatory cytokines (IL-1 $\beta$, IL- 6 and TNF- $\alpha$ ) caused by Lipopolysaccharides (LPS). In our previous study, mice spleen was used to detected the mechanism of PAMK, and find that PAMK could enhance cytokines secretion by stimulating the TLR4/MyD88/NF-KB signaling pathway ${ }^{8}$. However, the mechanism of PAMK in regulating the immune function of macrophages was still not clear. Therefore, RAW264.7 was used in this experiment.

TLR4 plays an important role in immunomodulatory, which could recognize the pathogen-related molecular patterns, transmit signal molecules to the cytoplasm, and promote the production of proinflammatory factors ${ }^{10}$. There are two ways to active the TLR4/NF-kB signal pathway: the dependent of myeloiddifferentiationfactor8 (MyD88) signal pathway and independent of MyD88 signal pathway. When TLR4 was active, the intracellular TIR region binds to the carboxyl group of MyD88, and its amino group binds to IL-1 amino group to activate IL-1 receptor-associated kinase (IRAK), IRAK activates TNF-receptorassociated factor6 (TRAF6), TRAF6 activates inhibitor of NF-KB kinases (IKKs), and the NF-KB inhibitor phosphorylates and degrades under the action of IKKs compound to activates NF-KB, then induces the expression of specific cytokines (IL-1,IL-6, IL-8, IL-12) in the nucleus ${ }^{11}$. As some article suggested that 
polysaccharide regulate the immune function through TLR4 signal pathway. Some researchers indicated that a novel polysaccharide obtained from Craterellus cornucopioides, polysaccharides from Cordyceps gunnii mycelia, polysaccharide from wheat bran could induce lymphocyte proliferation, promote macrophage activation and phagocytic activity, promote cytokines IL-2, IL-6, IL-1 $\beta$, TNF- $\alpha$ and IFN- $\gamma$ secretion and increase immune function through TLR4 signal pathway ${ }^{12-14}$.

Based on previous research, we preliminary confirmed that PAMK promote cytokines secretion by stimulating TLR4/MyD88/NF-KB signal pathway in mice spleen. Thus, we chose RAW264.7 as immune cell model in vitro, and measured serum cytokines of cellular supernatant, mRNA relative expression of cytokines and TLR4 signal pathway, protein expression level of TLR4 signal pathway by ELISA, qPCR, and western blot to further confirm that PAMK play an important role in promote the cytokines through TLR4 signal pathway in RAW264.7.

\section{Results}

\subsection{The effects of different TLR4-siRNAs on TLR4 mRNA relative expression}

Total RNA was extracted from RAW 264.7 to measure the mRNA relative expression of TLR4. As presented in Fig. 1, the results showed that si-TLR4-2 at $100 \mathrm{nM}$ has the best inhibitory effect and efficency of inhibiting TLR4 expression was up to $72 \%$.

PAMK promoted the cytokines production of RAW264.7 cellular supernatant

The cellular supernatant of six groups was collected, and then measure cellular supernatant cytokines level. The results in Fig. 2A-Figure2D revealed that all the cytokines were significantly $(P<0.05)$ down regulated by siTLR4 when compared with NC group. cellular supernatant cytokines levels in cell group and NC group showed no significant difference, and PAMK group and NC + PAMK group showed no significant difference $(P>0.05)$, which means that lip 3000 has no toxic side effect to RAW264.7. However, the cellular supernatant of all cytokines treated with PAMK were significantly $(P<0.05)$ upregulated when compared with siTLR4 group, which indicated that PAMK could promote cellular supernatant cytokines production.

\subsection{PAMK promoted the mRNA relative expression of cytokines}

Total RNA was extracted from RAW 264.7 to measure the mRNA relative expression of cytokines. Four genes were tested by qPCR. As showed in the Fig. $2 \mathrm{E}$-Figure $2 \mathrm{H}$, the results indicated that the relative mRNA of IL-2, IL-4, TNF-a, and IFN- $y$ in siTLR4 was significantly $(P<0.05)$ decreased when compared with NC group. The relative mRNA expressions of TNF-a, and IFN- $\gamma$ were significantly $(P<0.05)$ upregulated and IL-2, IL-4 had the uptrend in siTLR4 + PAMK group compared to siTLR4 group. Compared with cell 
group and NC + PAMK group respectively, the relative mRNA expression showed no significant $(P>0.05)$ difference in NC group and PAMK group. All the results indicated that PAMK could promote relative mRNA of cytokines expression production.

\subsection{PAMK promoted the relative mRNA expression of TLR4 signaling pathway.}

Total RNA was extracted from RAW 264.7 to measure the relative mRNA expression of TLR4 signal pathway. The results were showed in Fig. 3. The relative mRNA expressions of TLR4, MyD88, NF-KB, IKK were significantly $(P<0.05)$ down regulated in siTLR4 group compared with NC group. However, when treating with PAMK, NF-KB significant $(P<0.05)$ increased and TLR4, MyD88 and IKK had the uptrend. The difference between cell group and NC group, NC + PAMK group and PAMK group has no significant difference $(P>0.05)$. Thus, the results indicated that PAMK could promote relative mRNA expression of TLR4 signal pathway.

\subsection{PAMK regulated the relative protein expression in the TLR4 signaling pathway}

Proteins were extracted from RAW 264.7 to measure the relative protein level of NF-KB, TLR4, MyD88, IKK, GAPDH. The relative protein expression level of the genes were showed in Fig. 4. The results indicated that in siTLR4 group, the relative protein expression level of all genes were significantly $(P<0.05)$ increased than NC group. The relative protein expression of TLR4 was significant upregulated $(P<0.05)$ and NF-KB, MyD88, IKK has the uptrend in siTLR4 + PAMK group compared with siTLR4 group. The relative protein expression level has the same trend with the relative mRNA expression level. The above results suggested that PAMK could regulate the protein expression level in the TLR4 signaling pathway.

\section{Discussion}

PAMK has the function of promoting the lymphocytes active and proliferation, maintaining the stable cell morphology, increasing the secretion of cytokines such as TNF-a, IL-4, IL-6, IL-10, IL-17, IL-17A, IFN-y, alleviating the immunosuppression caused by CTX, and increasing the index of immune organs, which was considered one of the characteristic of the immune level enhance ${ }^{15} ;{ }^{16}$. We all know that cytokines play a very important role in body immunity ${ }^{15} ;{ }^{17} ;{ }^{18}$ and macrophages play an important role in defending the body against invading pathogens ${ }^{15} ;{ }^{17} ;{ }^{18}$. Increased level of IL-4, IL-2, TNF-a and IFN-Y could enhance the macrophage activity and improve specific immunity. In our research, we found that PAMK could upregulated the mRNA and protein expression of IL-2, IL-4, TNF- $a$ and IFN- $\gamma$ caused by siTLR4. IL-2 can enhance the secretion of TNF-a, IL-1, NO and other cytokines from macrophages, enhance the cytotoxic effect of macrophages and increase the killing activity of macrophages. IL-4 can increase the tumor killing ability of bone marrow $M \varphi$ and the expression of $\mathrm{Fc}(\mathrm{II})$ receptor, and promote the transformation of macrophages from $M 1$ to $M 2$ type. From our research, the results indicated that the relative mRNA expression level andcellular supernatant level of IL-2, IL-4 were significant decreased by 
siTLR4, but it significant increased or had increase trend after PAMK treated. Thus, the proliferative activity of macrophages is also increased, and the secretion levels of TNF- $\alpha$ and IFN- $\gamma$ also increased when treated with PAMK, just as our research showed. According, we suggested that PAMK could enhanced macrophage activity and enhanced the release of TNF- $a$ and IFN- $\gamma$ to increased macrophage immunomodulation. S, Li, Shi etc found polysaccharide from Auricularia auricula, fungal polysaccharide could promoted the activation of macrophages and induced the secretion of cytokines such as IL-1 $\beta$, IL-6, NO and TNF-a to enhance the phagocytic activity of macrophages and increase macrophage immunomodulation ${ }^{19} ;{ }^{21} ;{ }^{22}$. However, Zeng, Ma.G, Wang etc indicated that Lonicerae Japonicae Flos and Lonicerae Flos, polysaccharide from Pleurotus eryngii and Procyanidin A2 could inhibit of NO secretion in RAW264.7, down-regulate of IL-1 $\beta$, IL- 6 and TNF-a release, down-regulate of iNOS protein and COX2, NF$K B(p-I K K a / \beta, p-I K B a$ and $p-p 65)$ and MAPK (p-p38, p-JNK and p-ERK) protein levels caused by LPS ${ }^{23-25}$. These results were similar to our research. Consequently, we suggested that PAMK could maintain stable immune levels of macrophages and maintain the body's immune balance when the body is stimulated by either LPS or siTLR4, etc.

TLR4, an important member of the TLR family, was the first upstream gene for NF-kappaB (NF-kB) and has been shown to be involved in immune responses ${ }^{26}$. Some research suggested that when TLR4 is activated, there are two conduction signal pathway: dependent myeloiddifferentiationfactor88 (MyD88) signaling pathway and non-dependent MyD88 signaling pathway. The activated TLR domain of TLR4 binds to PAMK in a complex that can bind to MyD88. Interleukin-1 receptor associated kinase 1 (IRAK1), IRAK4 and TNF receptor associated factor 6 (TRAF6) recruit to the TLR-MyD88 complex, resulting in phosphorylation of IRAK1 and TRAF6. The signal is propagated by the phosphorylated linker molecular complex to downstream MAP kinases (JNK, p38 MAPK), Apetala 1 (AP1) and the nuclear factor kappa-B kinase (IKK) complex NF-KB. Activation of inhibitor of IKKa/ $\beta$ phosphorylates inhibitor of NF-KB (IKB), which is shed from NF-KB and ubiquitinated, allowing NF-KB to be activated by the inhibitory state leading to transcription factor activation ${ }^{12} ;{ }^{27} ;{ }^{28}$. In our research, we indicated that PAMK could increase the relative mRNA expression level and protein expression level of TLR4, MyD88, IKKa/ $\beta$, NF-KB in siTLR4 + PAMK group when it compared with siTLR4 group, and we found that the tend of mRNA and protein level was the same. Thus, We suggested that PAMK activate IKK through a MyD88-dependent pathway, which phosphorylates IKB and make it off NF-KB and allow NF-KB to enter the nucleus to exert immunoregulatory effects. When NF-KB signal pathway was activated, cells would secrete cytokines to maintain the immunity balance while the organism is stimulated, such as IL-2, IL-4, TNF-a and IFNas our research showed. Li indicated that PAMK could increase the serum level of IL-2, IL-4, TNF-a and IFN- $\gamma$, enhance splenocytes immunomodulatoryactivity by TLR4/MyD88/NF-kB signal pathway ${ }^{9}$. Some researchers showed that Polysaccharides from Cordyceps gunnii mycelia could increase the spleen thymus index, up-regulate the serum level of IL-2, IL-12, IFN-y, IgG and other cytokines secretion levels, protect immunosuppressed mice through TLR4/TRAF6/NF-KB signaling pathway ${ }^{29}$. These findings were consistent with previous research. However, Zheng, He and Jun suggested that Diethyl Blechnic Exhibits, an inulin-type fructan from Codonopsis pilosula, a novel polysaccharide from Acorus tatarinowii could inhibit release of pro-inflammatory factors caused by LPS such as prostaglandin E2 (PGE2) and relative 
mRNA expression of TNF-a, IL-1 $\beta$, IL- 6 and myeloperoxidase (MPO) through TLR4/MyD88/NF-KB in RAW264.7 $7^{30-33}$. That also indicated that polysaccharide regulate RAW264.7 immune function by TLR4/MyD88/NF-KB signal pathway.

In conclusion, our research indicated that PAMK could increase the relative mRNA expression level and serum cytokines level of IL-2, IL-4, TNF- $a$ and IFN- $\gamma$, enhance the immunomodulatory activity of RAW264.7 by TLR4/MyD88/NF-KB signal pathway

\section{Methods}

\subsection{Culture, grouping and transfection of RAW264.7}

PAMK (purity $95 \%$ ) was purchased from Yanglingciyuan Biotechnology company (Xi'an, China). PAMK was diluted to $12.5 \mu \mathrm{g} / \mathrm{mL}$ with RAW264.7 culture medium (Procell Life Science Technology Co.,Ltd.,Wuhan, Chian). Mice mononuclear macrophage leukemia cells RAW264.7 (Zhongqiao Xinzhou Biotechnology Co., Ltd, Shanghai, China), adjusted to a density of $1 \times 10^{6}$ cells $/ \mathrm{mL}$, and cultured in a 12well plate (Corning Incorporated, USA). After $4 \mathrm{~h}$ of culture, randomly devided into six groups (cell, NC, siTLR4, NC + PAMK, PAMK, siTLR4 + PAMK). Transfection reagent NC, siTLR4 (RiboBio Co., LTD, Guangzhou, China), (Table 1), and PAMK was added, and cells were collected $24 \mathrm{~h}$ later for mRNA relative expression detection.

Table 1

Sequences for siTLR4

\begin{tabular}{|l|lll|}
\hline cDNA & Primer sequences & cDNA & Primer sequences \\
\hline si-TLR4-1 & CAATTCTGTTGCTTGTATA & si-TLR4-3 & CCATGAACTGACTCTAAGA \\
\hline si-TLR4-2 & GTCCCTGATGACATTCCT & \\
\hline
\end{tabular}

Total RNA was extracted from the RAW264.7 with TRIzol reagent (Thermo Fisher, USA) and reversetranscribed was performed by the Reverse Transcription Kit (Takara, Japan). The primers (Table 2) of related genes were designed by using Primer Premier 5.0 software (Premier Biosoft International, USA). An ABI PRISM 7500 detection system (Applied Biosystems, Foster City, CA) was used to measure the mRNA relative expression of genes. 
Table 2

Primer sequences for qPCR

\begin{tabular}{|llll|}
\hline cDNA & Primer sequences & cDNA & Primer sequences \\
\hline TLR4-F & ACCTGGAATGGGAGGACAA & TLR4-R & GTCCAAGTTGCCGTTTCTTG \\
\hline -actin-F & GCTTCTAGGCGGACTGTTAC & $\beta$-actin-R & CCATGCCAATGTTGTCTCTT \\
\hline MyD88-F & GGCAACTAGAACAGACAGA & MyD88-R & GTGCAAGGGTTGGTATAGTC \\
\hline IKK-F & TGCAGCTGATGCTTATGTG & IKK-R & TGCCTGTGACCATGTTCAA \\
\hline NFKB-F & GAACCAGGGTGTGTCCATG & NFKB-R & TCCGCAATGGAGGAGAAGTC \\
\hline IL2-F & CCTGAGCAGGATGGAGAAT & IL2-R & CGCAGAGGTCCAAGTTCAT \\
\hline IL4-F & GAGATGGATGTGCCAAACG & IL4-R & AGCACCTTGGAAGCCCTACA \\
\hline IFN-Y-F & GGCTTCCATCATGAACAAC & IFN-Y-R & TGTAGGTGAGGTTGATCTTTC \\
\hline TNF-a-F & TCGAGGCTCCAGTGAATT & TNF-a-R & GCTTCTAGGCGGACTGTTAC \\
\hline
\end{tabular}

\subsection{Assays of cell cytokines in cellular supernatant}

The cellular supernatant of six groups was collected.. The levels of IL-2 (70-EK2022/2), IL-4 (70EK2042/2), IFN-ץ (70-EK2802/2), and TNF-a (70-EK2822/2) were measured by using ELISA kits (Hangzhou Lianke Biotechnology Co., Ltd., Hangzhou, China).

\subsection{Western blot analysis}

The total protein was extracted by RIPA (Biyuntian Biological Technology Co., Ltd., Shanghai, China), adjusted the concentration with $5 \times$ simple buffer (Biyuntian Biological Technology Co., Ltd., Shanghai, China), and denatured by heating at $100{ }^{\circ} \mathrm{C}$ for $10 \mathrm{~min}$. We analyzed it by sodium dodecyl sulfate polyacrylamide gel electrophoresis (SDS-PAGE) under reducing conditio $12 \%$ gels were used to transfer to PVDF membranes using a tank transfer system at $200 \mathrm{~mA}$ in Tris-glycine buffer containing $20 \%$ methanol for $70-90$ min at $4{ }^{\circ} \mathrm{C}$. The membranes were then blocked with $5 \%$ skim milk at $37^{\circ} \mathrm{C}$ for 60 min. Next, the membranes were incubated with diluted primary antibodies overnight; the diluted concentrations and antibody sources are shown in Table 3. We incubated peroxidase-conjugated secondary antibodies against rabbit IgG at room temperature for $1 \mathrm{~h}(1: 10000$, Beijing Boaosen Biotechnology Co., Ltd, China). Chemiluminescence Detection Reagents (Applygen, Beijing) were incubated for $1 \mathrm{~min}$ to determine the ratios of target protein signals on a Chemiluminescence Imaging System (Tianneng, Shanghai) to signals of control proteins. GAPDH (Abcam) served as internal controls, and the relative expression levels of the proteins were expressed as the ratios of gray value of each of these proteins to that of GAPDH. 
Table 3

Diluted concentrations and antibody sources

\begin{tabular}{|lll|}
\hline Antibody & Antibody sources & Antibody dilited \\
\hline TLR4 & AF7017 & $1: 1000$ \\
\hline MyD88 & AF5195 & $1: 1000$ \\
\hline IKK & AF6014 & $1: 2000$ \\
\hline NF-KB & AF5006 & $1: 2000$ \\
\hline GAPDH & Abcam9484 & $1: 1000$ \\
\hline
\end{tabular}

\subsection{Statistical analysis}

The data were analysed using SPSS for Windows (version 18, SPSS Inc, Chicago, IL, USA) and expressed as the means \pm standard deviations (SD). All the qPCR assays were repeated in triplicate, and the relative expression levels were measured in terms of threshold cycle $(\mathrm{Ct})$ values and normalized via the formula $2^{-\Delta \Delta C t}$. Differences between groups were compared using one-way analysis of variance (ANOVA) followed by Tukey's honestly significant difference test. $P<0.05$ was considered statistically significant.

\section{Declarations}

\section{Conflict of interest statement}

There is no conflict of interest in this manuscript.The content has not been published or submitted for publication elsewhere.All authors are in agreement with the content of the manuscript and submission in this journal.

\section{Acknowledgement}

Wanyan Li contributed to the hypothesis generation. Yunbo Tian contributed to experimental design. Wenbin Chen contributed to data interpretation. Xumeng Zhang contributed to manuscript preparation. Xuelian Xiang conducted the experiments. Nan Cao contributed to the data interpretation. Danning Xu participated in the experiment. Wenjun Liu contributed to collection of experimental samples. Xinliang Fu was responsible for the purchase of related materials. Jia Liu contributed to data processing. All authors reviewed the manuscript.

\section{Founding}

This work was supported by Basic and Applied Basic Research Fund Project of Guangdong Province (2019A1515110106); Science and Technology Plan Project of Guangzhou (grant No.201904010076); Innovation fund support of Zhongkai College of Agricultural Engineering (grant No.2019A04). This funding had no impact on our study design or collection, analysis, and interpretation of the data. Further support was provided solely from institutional and/or departmental sources. 


\section{References}

1. Guo, L., Sun, Y. L., Wang, A. H., Xu, C. E. \& Zhang, M. Y. Effect of Polysaccharides Extract of Rhizoma Atractylodis Macrocephalae On Thymus, Spleen and Cardiac Indexes, Caspase-3 Activity Ratio, Smac/DIABLO and HtrA2/Omi Protein and mRNA Expression Levels in Aged Rats. Mol. Biol. Rep. 39, 9285-9290 (2012).

2. Xu, D. \& Tian, Y. Selenium and Polysaccharides of Atractylodes macrocephala Koidz Play Different Roles in Improving the Immune Response Induced by Heat Stress in Chickens. Biol. Trace Elem. Res. 168, 235-241 (2015).

3. Shi, Y. Y., Guan, S. H., Tang, R. N., Tao, S. J. \& Guo, D. A. Simultaneous Determination of Four Sesquiterpenoids in Atractylodes Macrocephala Rhizoma by GC-FID: Optimisation of an UltrasoundAssisted Extraction by Central Composite Design. Phytochem Anal. 23, 408-414 (2012).

4. Li, W. et al. Polysaccharide of Atractylodes macrocephala Koidz (PAMK) Relieves Immunosuppression in Cyclophosphamide-Treated Geese by Maintaining a Humoral and Cellular Immune Balance. Molecules.23, (2018).

5. Li, R., Sakwiwatkul, K., Yutao, L. \& Hu, S. Enhancement of the Immune Responses to Vaccination Against Foot-And-Mouth Disease in Mice by Oral Administration of an Extract Made From Rhizoma Atractylodis Macrocephalae (RAM). Vaccine. 27, 2094-2098 (2009).

6. Liu, J. et al. Effect of Selenylation Modification On Immune-Enhancing Activity of Atractylodes Macrocephala Polysaccharide. Int. J. Biol. Macromol. 72, 1435-1440 (2015).

7. Xu, D. et al. The Protective Effects of Polysaccharide of Atractylodes Macrocephala Koidz (PAMK) On the Chicken Spleen Under Heat Stress Via Antagonizing Apoptosis and Restoring the Immune Function. Oncotarget. 8, 70394-70405 (2017).

8. Jeong, D., Dong, G. Z., Lee, H. J. \& Ryu, J. H. Atractylodes macrocephalaAnti-Inflammatory Compounds From. Molecules (Basel, Switzerland). 24, (2019).

9. Li, B. X. et al. Atractylodes macrocephalaPolysaccharide of Koidz Enhances Cytokine Secretion by Stimulating the Signaling Pathway in the Mouse Spleen. J. Med. Food. 22, 937-943 (2019).

10. Xu, Z. et al. Immunomodulatory Mechanism of a Purified Polysaccharide Isolated From Isaria Cicadae Miquel On RAW264.7 Cells Via Activating TLR4-MAPK-NF-kappaB Signaling Pathway. Int. J. Biol. Macromol.(2020).

11. Liu, T. Y. et al. Polygonatum Sibiricum Polysaccharides Prevent LPS-induced Acute Lung Injury by Inhibiting Inflammation Via the TLR4/Myd88/NF-кB Pathway. Exp. Ther. Med. 20, 3733-3739 (2020).

12. Guo, M. Z., Meng, M., Feng, C. C., Wang, X. \& Wang, C. L. A Novel Polysaccharide Obtained From Craterellus Cornucopioides Enhances Immunomodulatory Activity in Immunosuppressive Mice Models Via Regulation of the TLR4-NF-kB Pathway. Food Funct. 10, 4792-4801 (2019).

13. Wang, H. et al. A Homogalacturonan From Hippophae Rhamnoides L. Berries Enhance Immunomodulatory Activity through TLR4/MyD88 Pathway Mediated Activation of Macrophages. 
Int. J. Biol. Macromol. 107, 1039-1045 (2018).

14. Shen, T. et al. Polysaccharide From Wheat Bran Induces Cytokine Expression Via the Toll-Like Receptor 4-Mediated P38 MAPK Signaling Pathway and Prevents Cyclophosphamide-Induced Immunosuppression in Mice. Food Nutr. Res. 61, 1344523 (2017).

15. Hu, J. F., Zhang, W., Zuo, W., Tan, H. Q. \& Bai, W. Inhibition of the PD-1/PD-L1 Signaling Pathway Enhances Innate Immune Response of Alveolar Macrophages to Mycobacterium Tuberculosis in Mice. Pulm. Pharmacol. Ther. 60, 101842 (2020).

16. He, W., Li, X., Peng, Y., He, X. \& Pan, S. Anti-Oxidant and Anti-Melanogenic Properties of Essential Oil From Peel of Pomelo Cv. Guan Xi.Molecules (Basel, Switzerland). 24, (2019).

17. Jin, M. et al. Effects of Polysaccharide From Mycelia of Ganoderma Lucidum On Intestinal Barrier Functions of Rats. Int. J. Biol. Macromol. 94, 1-9 (2017).

18. Li, G., Xiang, Y., Zhao, J. \& Chang, J. In vitroSaccharum Alhagi Polysaccharide-1 and - 2 Promote the Immunocompetence of RAW264.7 Macrophages. Exp. Ther. Med. 15, 3556-3562 (2018).

19. Hu, J. F., Zhang, W., Zuo, W., Tan, H. Q. \& Bai, W. Inhibition of the PD-1/PD-L1 Signaling Pathway Enhances Innate Immune Response of Alveolar Macrophages to Mycobacterium Tuberculosis in Mice. Pulm. Pharmacol. Ther. 60, 101842 (2020).

20. Zhao, Q. et al. Norcantharidin Facilitates LPS-mediated Immune Responses by Up-Regulation of AKT/NF-kappaB Signaling in Macrophages. PLoS One. 7, e44956 (2012).

21. Li, H., Xie, W., Sun, H., Cao, K. \& Yang, X. Effect of the Structural Characterization of the Fungal Polysaccharides On their Immunomodulatory Activity.Int. J. Biol. Macromol.(2020).

22. Shi, F. et al. Comparison of Physicochemical Characteristics and Macrophage Immunostimulatory Activities of Polysaccharides From Chlamys Farreri.Mar. Drugs.18, (2020).

23. Ma, G. et al. Preparation of Newly Identified Polysaccharide From Pleurotus Eryngii and its AntiInflammation Activities Potential. J. Food Sci. 85, 2822-2831 (2020).

24. Zeng, A. Q. et al. [Comparative Study On Anti-Inflammatory Effect of Lonicerae Japonicae Flos and Lonicerae Flos]. Zhongguo Zhong Yao Za Zhi. 45, 3938-3944 (2020).

25. Wang, Q. Q. et al. Procyanidin A2, a Polyphenolic Compound, Exerts Anti-Inflammatory and AntiOxidative Activity in Lipopolysaccharide-Stimulated RAW264.7 Cells. PLoS One. 15, e237017 (2020).

26. Biemmi, V. et al. Inflammatory Extracellular Vesicles Prompt Heart Dysfunction Via TRL4-dependent NF-кB Activation. Theranostics. 10, 2773-2790 (2020).

27. Xu, W. et al. Signaling Pathway Underlying Splenocytes Activation by Polysaccharides From Atractylodis Macrocephalae Koidz. Mol. Immunol. 111, 19-26 (2019).

28. He, L. et al. Sinomenine Down-Regulates TLR4/TRAF6 Expression and Attenuates Lipopolysaccharide-Induced Osteoclastogenesis and Osteolysis. Eur. J. Pharmacol. 779, 66-79 (2016).

29. Meng, M., Wang, H., Li, Z., Guo, M. \& Hou, L. Protective Effects of Polysaccharides From Cordyceps Gunnii Mycelia Against Cyclophosphamide-Induced Immunosuppression to TLR4/TRAF6/NF- 
kappaB Signalling in BALB/c Mice. Food Funct. 10, 3262-3271 (2019).

30. Ju, M. et al. MicroRNA-27a Alleviates LPS-induced Acute Lung Injury in Mice Via Inhibiting Inflammation and Apoptosis through Modulating TLR4/MyD88/NF-KB Pathway. Cell cycle (Georgetown, Tex.). 17, 2001-2018 (2018).

31. He, J. et al. Diethyl Blechnic Exhibits Anti-Inflammatory and Antioxidative Activity Via the TLR4/MyD88 Signaling Pathway in LPS-Stimulated RAW264.7 Cells.Molecules (Basel, Switzerland). 24, (2019).

32. Meng, Y. et al. Extraction, Characterization and Anti-Inflammatory Activities of an Inulin-Type Fructan From Codonopsis Pilosula. Int. J. Biol. Macromol. 163, 1677-1686 (2020).

33. Zhong, J., Qiu, X., Yu, Q., Chen, H. \& Yan, C. A. Novel Polysaccharide From Acorus Tatarinowii Protects Against LPS-induced Neuroinflammation and Neurotoxicity by Inhibiting TLR4-mediated MyD88/NFkappaB and PI3K/Akt Signaling Pathways. Int. J. Biol. Macromol. 163, 464-475 (2020).

\section{Figures}
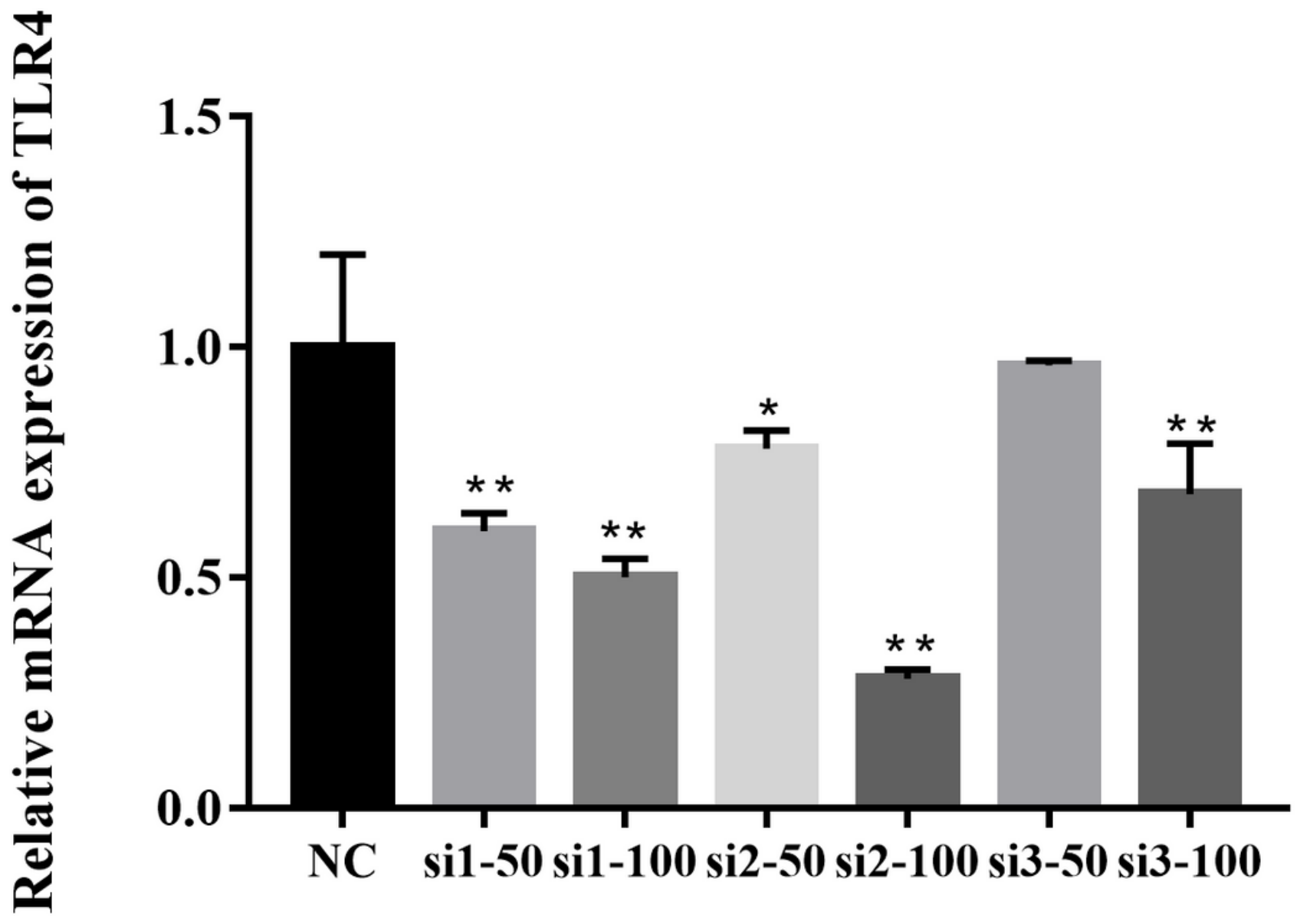

Figure 1 
The effects of different TLR4-siRNAs on TLR4 mRNA relative expression. Data were expressed as the means $\pm S D, n=3 .{ }^{*} P<0.05, * * P<0.01$, compared with the NC group. .

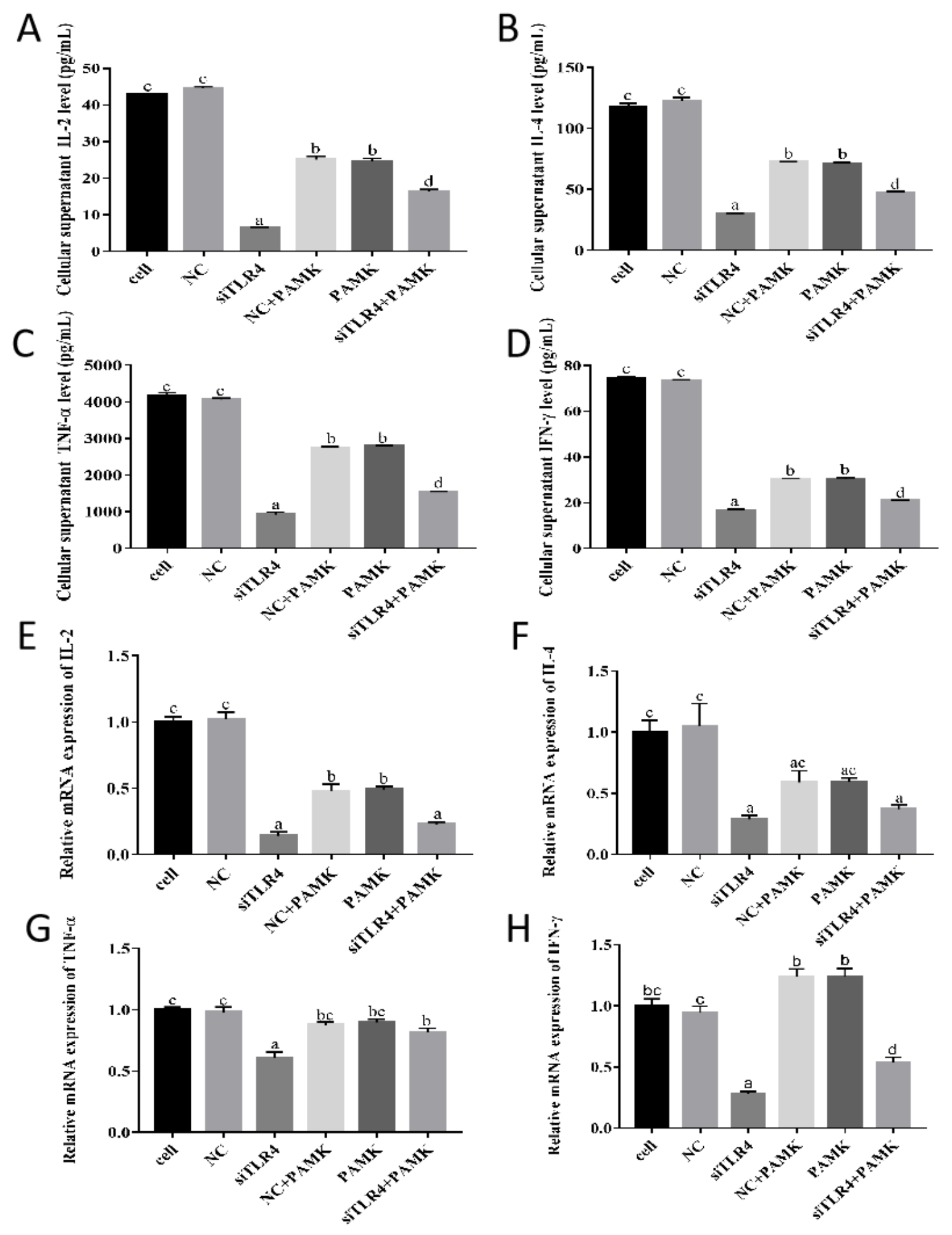

Figure 2

Effects of PAMK on cellular supernatant and relative mRNA expression level of cytokines in mice RAW 264.7 treated with siTLR4. Cellular supernatant cytokines levels of (A) IL-2; (B) IL-4; (C) TNF-a; (D) IFN-ץ; Relative mRNA expression of (E) IL-2; (F) IL-4; (G) TNF-a; (H) IFN-y; Data were expressed as the means \pm 
$S D, n=6$. Means with different letters represent statistically significant difference $(P<0.05)$; the bars with the same letter are not significantly difference $(P>0.05)$.
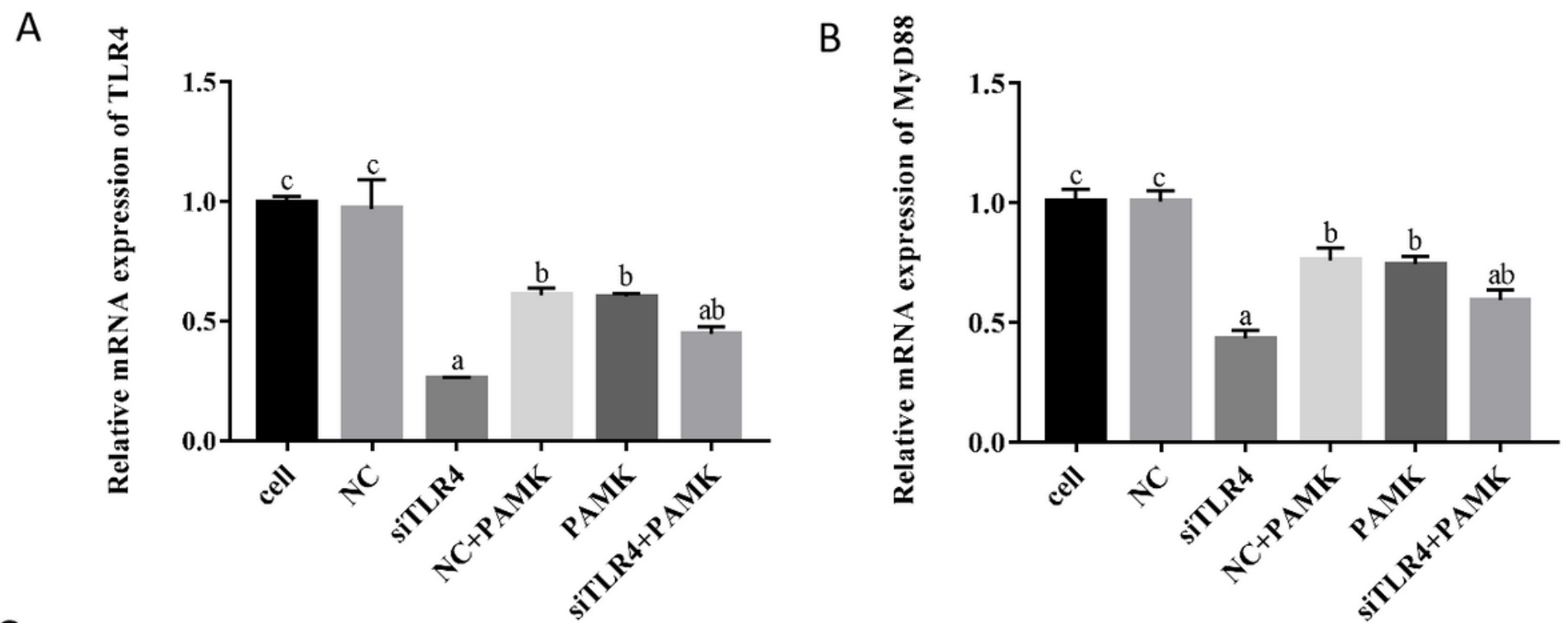

C
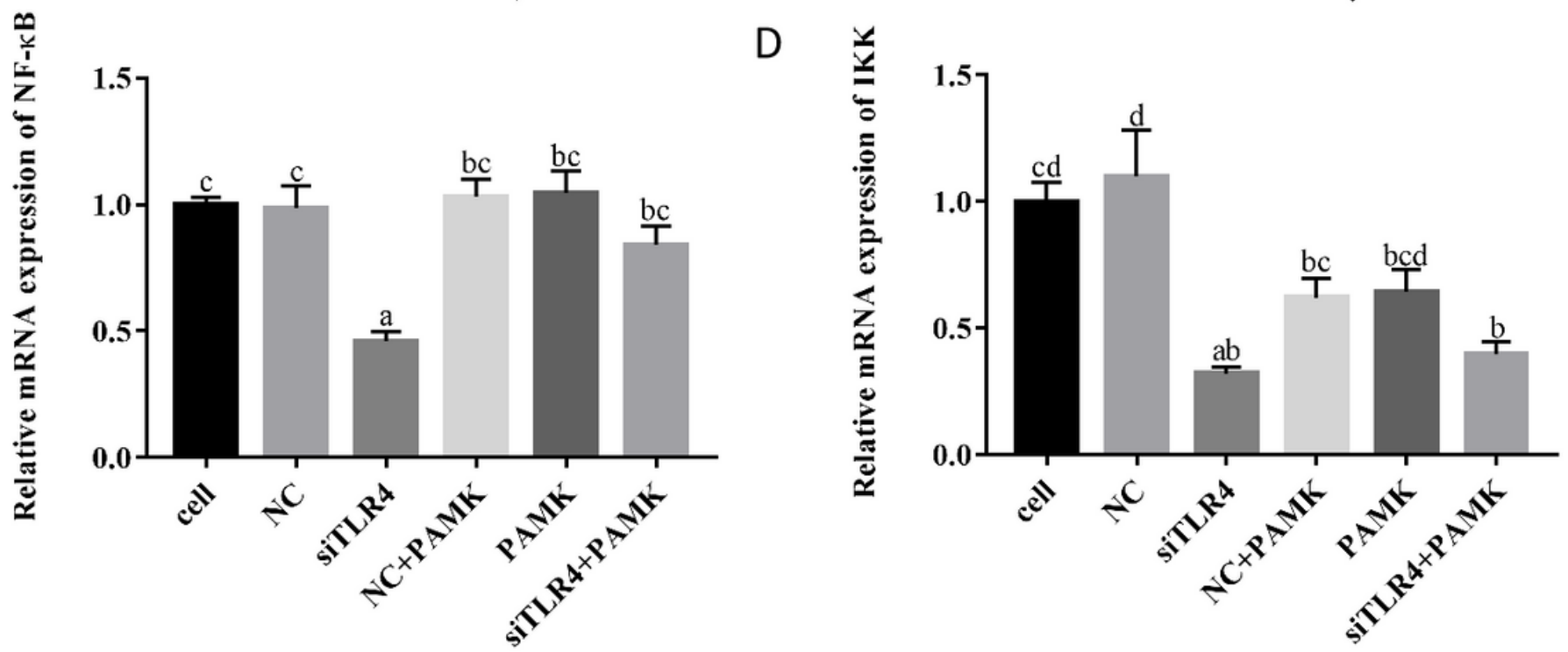

Figure 3

Effects of PAMK on the TLR4 signal pathway in mice RAW264.7 treated with siTLR4. Relative mRNA expression of (A) TLR4; (B) MyD88; (C) NF-KB; (D) IKK; Data were expressed as the means \pm SD, $n=6$. Data were expressed as the means $\pm S D, n=6$. Means with different letters represent statistically significant difference $(P<0.05)$; the bars with the same letter are not significantly difference $(P>0.05)$. 


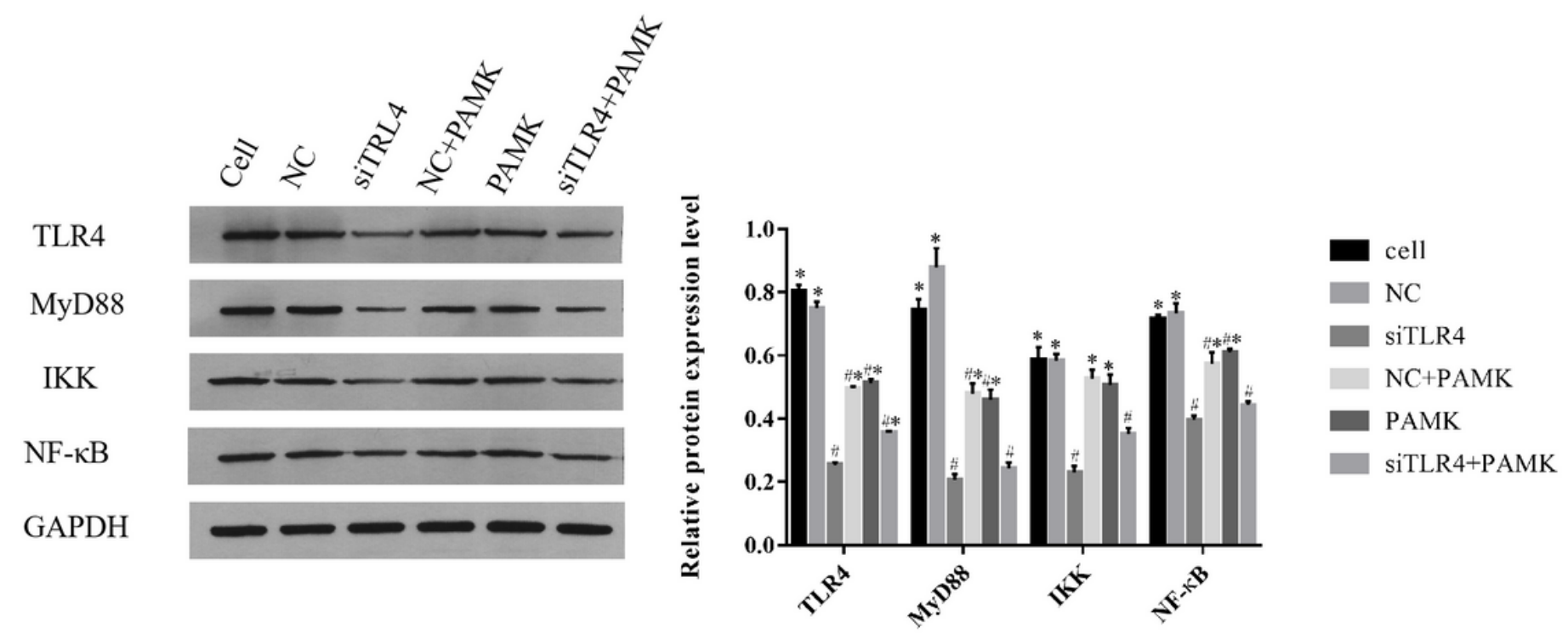

Figure 4

Relative protein level. Data were presented as the means $\pm S D, n=3$. \#P $<0.05$, compared with the NC group; ${ }^{*} \mathrm{P}<0.05$, compared with the siTLR4 group.

\section{Supplementary Files}

This is a list of supplementary files associated with this preprint. Click to download.

- Supplementarymaterial.pdf 